\title{
PHOTOGRAMMETRIC TRACKING OF AERODYNAMIC SURFACES AND AEROSPACE MODELS AT NASA LANGLEY RESEARCH CENTER
}

\author{
Mark R. Shortis $^{\mathrm{a}^{*}}$, Stuart Robson ${ }^{\mathrm{b}}$, Thomas W. Jones ${ }^{\mathrm{c}}$, William K. Goad ${ }^{\mathrm{d}}$, Charles B. Lunsford ${ }^{\mathrm{c}}$ \\ a School of Science, RMIT University, \\ GPO Box 2476, Melbourne 3001, Australia. mark.shortis@rmit.edu.au \\ ${ }^{\mathrm{b}}$ Department of Civil, Environmental and Geomatic Engineering, University College London, \\ Gower Street, London WC1E 6BT, U.K. s.robson@ucl.ac.uk \\ c Advanced Sensing and Optical Measurement Branch, NASA Langley Research Center, \\ Hampton VA 23681, U.S.A. thomas.w.jones@nasa.gov charles.b.lunsford@nasa.gov \\ ${ }^{\mathrm{d}}$ National Transonic Facility, NASA Langley Research Center, \\ Hampton VA 23681, U.S.A. william.k.goad@nasa.gov
}

Commission V, WG V/1

KEY WORDS: Photogrammetry, tracking, measurement, sequence, surface, shape, model, 6DoF

\begin{abstract}
:
Aerospace engineers require measurements of the shape of aerodynamic surfaces and the six degree of freedom (6DoF) position and orientation of aerospace models to analyse structural dynamics and aerodynamic forces. The measurement technique must be noncontact, accurate, reliable, have a high sample rate and preferably be non-intrusive. Close range photogrammetry based on multiple, synchronised, commercial-off-the-shelf digital cameras can supply surface shape and 6DoF data at $5-15 \mathrm{~Hz}$ with customisable accuracies. This paper describes data acquisition systems designed and implemented at NASA Langley Research Center to capture surface shapes and 6DoF data. System calibration and data processing techniques are discussed. Examples of experiments and data outputs are described.
\end{abstract}

\section{INTRODUCTION}

NASA Langley Research Center (LaRC) in Virginia specialises in aerodynamic testing and aerospace technology. Two of the principal experimental programs at LaRC are the design and analysis of aerodynamic structures and aerospace models. Research programs include both internal research projects and external consulting for civilian and military organisations. Consequently there is a continual need for non-contact, accurate, reliable, non-intrusive measurement for laboratory testing of aerodynamic structures and wind tunnel testing of aerospace models.

The use of camera systems to record laboratory and wind tunnel experiments is widespread at LaRC, for both qualitative and quantitative analyses. The range of cameras includes every possible configuration, from single camcorders through to multiple digital cameras. Applications requiring image measurement include flow visualisation, radiometric analysis of pressure sensitive surfaces, model tracking, surface shape measurement and deformation monitoring. The principle advantages of imaging techniques in this context are flexibility, customisable accuracy, the non-contact nature of the measurement and the ability to archive and post-analyse long image sequences from experimental testing.

In the category of surface characterisation, many experiments have been conducted at LaRC to support dynamic testing of thin film and membrane surfaces. Thin films and membranes are used for aerodynamic applications such as stretched lens concentrators for solar arrays and the wings of micro-flight vehicles (Shortis et al., 2002). The main focus with these thin films and membranes is tracking the shape of the dynamic surfaces under induced vibration. Motions are typically cyclic and the accuracy requirements are not demanding, so the surfaces can be recorded and analysed using short stereo image sequences. As with many applications of surface characterisation, the data is presented as a visualisation of the motion and the numerical data is used in stress and strain analysis (Shortis et al., 2002).

Flexible surfaces such as parachutes have also been the subject of video measurement at LaRC (Shortis et al., 2007). Experimental testing to measure parachute shape during the inflation and decent phases can provide vital information for the quantitative assessment of parachute performance. Testing at LaRC has concentrated on parachute shape and tracking of the leading edge using stereo cameras systems deployed within parachute payloads for controlled drops indoors and from an outdoor gantry, followed by operational parachute drops from a helicopter by the US Army (Shortis et al., 2007).

Light, flexible membranes also have many aerospace applications, such as solar sails, sun shields, inflatable communications antennae, and inflatable solar arrays, solar power concentrators and transmitters (Pappa et al., 2001, 2002). The aerospace structures characteristically contain large areas of delicate membrane surface, up to tens or even hundreds of meters in size. The structures are compactly folded to be carried into space on existing launch vehicles, then deployed to many times their launch size. Testing at LaRC has included both static and dynamic tests on samples of membrane, inflatable 
support structures and full size communications antennae. Different materials and surface types have been tested, such as matte finishes for general purpose applications and aluminized kapton specifically for solar sails. Passive, retro-reflective, projected and laser illuminated fluorescent targets have all been used to define the surfaces to be measured (Pappa et al., 2003).

However the longest association between LaRC and image measurement is the use of camera systems for wind tunnel testing. LaRC was a pioneer in the use of camera systems to measure wing deflections and track the location and orientation of aerospace models, usually known as the 6 Degree of Freedom (6DoF) solution, in wind tunnels (Burner et al., 1985; Shortis and Snow, 1997; Barrows, 2007).

The use of photogrammetry for wind tunnel testing commenced at LaRC in the late 1970s with synchronous stereo-photography to measure deformations of aerospace models. Subsequent developments focussed on utilising video based systems both to provide access to hostile environments and to avoid tedious processing and measurement associated with long sequences of film based images. Vidicon tube type cameras and video tape recorders were adopted in the early 1980s (Burner et al., 1985) and successfully eliminated demands of the photographic processing and film inventory management. CCD cameras with analogue transmission (Shortis and Snow, 1997) and digital transmission (Shortis et al., 2007) followed in the 1990s and 2000s respectively, providing higher quality images and improved reliability. Most applications were not designed for real time capture, due to the limitations of band width and processing power, and instead image data was post-processed. Video streams or still image sequences were recorded to video tape or video disc, requiring synchronisation using injection of time codes into the images (Childers et al., 1994; Snow and Shortis, 1995), and resulting in increased noise and a loss of image integrity (Shortis et al., 1993).

$6 \mathrm{DoF}$ measurement in wind tunnels has made a significant contribution to the safety of air travel. Spin tunnel testing and wake vortex tracking are two examples of this contribution that have emanated from LaRC. Routine testing is necessary to characterise the spin modes and spin recovery for all aircraft, either at the design stage or once the aircraft is operational. Wind tunnel testing can also be used to assess the effect of aircraft modifications or various weapons loads, or suggest configuration changes to improve spin recovery. Video camera systems have been used to provide 6DoF data for model testing in the spin tunnel at LaRC since the 1990s (Snow et al., 1992; Shortis and Snow, 1997).

In the early 1990s a program of experiments to assess the impact of wing tip vortices on trailing aircraft was carried out using the 30 by 60 foot wind tunnel at LaRC (Childers et al., 1994, Shortis and Snow, 1997). Stereo-video cameras and retro-reflective targeting was used to track free flight models as they were flown through the vortex trails from a fixed generator wing. This research contributed to changes to flight rules and a minimum separation between aircraft during approach and landing at airfields (Cohen and Hockaday, 1998).

\section{MATERIALS AND METHODS}

\subsection{Transition to New Technology}

Technology is being continuously upgraded at LaRC. Like many organisations that use imaging systems for qualitative and quantitative measurements, and have an extensive test facility inventory, LaRC is in a constant state of transition across a range of different technologies and systems. Whilst many wind tunnel surveillance systems still use CCTV cameras with RS170 or NTSC video standards because of the simplicity, reliability and resistance to interference, imaging systems used for measurement have generally moved to digital transmission. CameraLink or IEEE-1394 Firewire, also known as i.Link, are the dominant choices of digital image capture. Camera Link and Firewire systems are very reliable but require specialised cables and connectors, have a limited cable length and depend on a compatible interface card in the PC. Despite the intended standardisation of CameraLink and Firewire, experience has shown that some cameras do not link seamlessly to compatible interface cards and the connection and synchronisation of large numbers of cameras is problematic.

The latest technology of digital transmission systems to become available is Gigabit Ethernet (GigE) video cameras. These cameras have a significant advantage in using standard Ethernet cables and thereby can communicate over long distances using existing data switches and routers. Power over Ethernet (PoE) compatible GigE cameras can be powered through the Ethernet connection using a suitable switch, but generally require a separate set of cables for synchronised capture of images. Notwithstanding these issues, wired Ethernet is pervasive in most facilities and both the utility and limitations of cables and switches are well understood. The implementation of a new systems based on IDS $\mu$ Eye miniature GigE cameras has been integrated with the development of two aerospace tracking projects described in Section 3.

In parallel with the changeover of camera equipment, advantage can be taken of greater communication bandwidth and PC computing capacity to move to real time, or near real time, 2D, $3 \mathrm{D}$ and $6 \mathrm{DoF}$ processing and data delivery. True real time image processing for multiple, synchronised cameras has required, until very recent times, specialised hardware and software that can be both expensive and application-specific (Wiora et al., 2004). Whilst there are more general purpose systems available from commercial vendors, nevertheless they tend to specialise in different tasks such as inspection ${ }^{1}$, motion capture $^{2}$ and optical CMMs using hand-held probes ${ }^{3}$.

However the combination of GigE cameras and current PC technology such as multi-core processors and graphics cards allows real time solutions, albeit at relatively modest frame rates, to be developed as a general solution that can be applied in different applications of image measurement. A new system, known as VMSCapture, has been developed using this technology base and will be described in Section 3.

\subsection{Targeting}

Well defined artificial targets to signalise points of interest or define the surface to be measured have been consistently used at LaRC for quantitative measurements. Passive, internally illuminated and retro-reflective targets have all been employed to define surface points in generally unfavourable circumstances (Figure 1). For wind tunnel applications in particular, the lack of control over ambient lighting is always a factor (Shortis and Snow, 1997). Often the primary reason for artificial targets is that the model surfaces lack any clearly defined features

\footnotetext{
${ }^{1}$ www.aicon3d.de, www.mapvision.fi

${ }^{2}$ www.optitrack.com, www.vicon.com

${ }^{3}$ www.metronor.com, www.gom.com
} 
because they are thin membranes or polished metal surfaces (Graves and Burner, 2001).

In addition to these issues, artificial targets are favoured for tracking applications to ensure a high level of accuracy of the motion, rotation or change of shape. The absolute position, orientation and shape of the surface, if required, may be derived from an initial, static test. The emphasis is typically on the accuracy of the relative changes in the surface to be tracked, in order to determine surface deformation or object motion. Although it is feasible to track natural surface features if they are available, it is generally accepted that artificial circular targets will always realise superior accuracy (Robson and Shortis, 1997).



Figure 1. Example of the impact of ambient lighting on measurement of passive wing targets in a wind tunnel.

\subsection{Camera Calibration}

The approach taken to camera calibration of multi-camera systems has been adapted to specific circumstances in the various wind tunnels, but the principles have been consistent. Wherever possible, cameras are calibrated in situ by introducing a known, reference target array into the test section during a wind off calibration process.

The calibration object should be a 3D target array to minimise the correlations between camera calibration and exterior orientation parameters. The object should be physically stable, at least for the duration of the image capture for the calibration. It should fill the fields of view of the cameras to ensure that camera calibration parameters, especially lens distortions, are accurately modelled across the entire image format. It should be rotated within the fields of view of the cameras for 20-40 frames captured to ensure that the photogrammetric network has high levels of numerical redundancy and an optimal intersection geometry at the targets. The cameras should be fixed in terms of location, focus, pan, tilt and zoom, and regardless of the approach to calibration, focus and zoom are generally locked using tape or set screws to minimise any potential change in the calibration. The conditions prevailing for the calibration should match, as nearly as possible, the conditions of the experiment in terms of ambient temperature in particular.

Unfortunately this ideal circumstance for the calibration and measurement is almost never realised in practice. Many of the wind tunnel test sections at LaRC are much larger than a conveniently portable test object. The object to be measured in the testing often occupies a large percentage of the fields of view of the cameras. Calibrations are generally only possible in wind off mode because of safety concerns, and the temperature during wind on testing can be substantially different, so the prevailing conditions for the calibration and measurement are generally disparate. In some cases the camera housings are built into removable walls or panels that are used to access the test section.

These limitations force a hybrid approach to camera calibration. Cameras are often pre-calibrated in the laboratory and rely on a consistent focus and zoom between calibration and measurement. The cameras may then be partially re-calibrated in the wind tunnel, often as part of the process of establishing the location and orientation of the cameras and the test object relative to the tunnel coordinate system. The parameters included in the partial re-calibration are dependent on the coverage within the fields of view, and may be limited only to the principal point location on the basis that a different orientation with respect to gravity may induce a change in the optical axis of the lens (Shortis et al., 1998). If the camera is in a housing and views the object through a port then re-calibration of the lens distortions will partly compensate for the refractive interfaces (Shortis, 2015).

An independent self-calibration network, usually conducted using a high resolution digital SLR or compact camera, is used to simultaneously establish a set of reference targets in the test section and determine the zero position of the aerospace model with respect to the tunnel coordinate system. The former is necessary to enable the determination of the location and orientation of the fixed cameras during the experimental testing. The resection of the cameras should, if possible, be a continuous process to counter any changes in the wind tunnel environment during wind on testing.

The zero position for the model is essential as a baseline for comparisons with the experimental data. For every captured set of images, the cameras are resected and the targets on the model are intersected, followed by a network solution if appropriate. The 6DoF solution and deformations such as wing deflections and wing twist are computed based on the differences in the target locations between the zero position and the model during the testing (Figure 2).

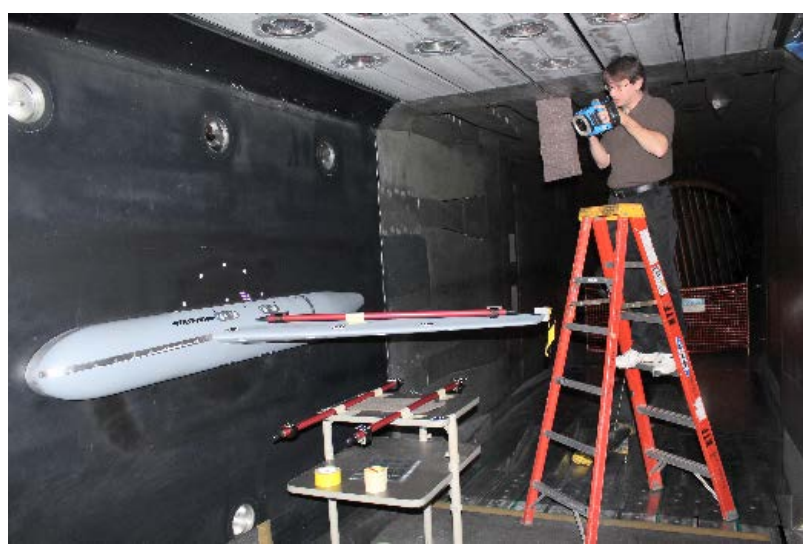

Figure 2. Test section set-up for the FastMac semi-span model in the NTF wind tunnel.

\section{AEROSPACE TRACKING APPLICATIONS}

\subsection{Microphone Array Tracking in the 14 by 22 Tunnel}

The 14 feet high by 22 feet wide $(4.3 \mathrm{~m}$ by $6.7 \mathrm{~m})$ subsonic tunnel $(14 \times 22)$ at LaRC is an atmospheric only, closed return tunnel that allows for a variety of test arrangements in a 50 foot 
(15.2 m) long test section. The test section can be closed (walls, ceiling and floor) or open (floor only) for versatility in the setup of sensors and instrumentation around the test section. The $14 \times 22$ tunnel is used primarily to evaluate the aircraft aerodynamic performance over a wide range of take off, cruise, landing and high angle-of-attack configurations.

Noise abatement has become an important consideration in aircraft design, with the primary intent of limiting the transmission of engine noise to the ground. However, the noise generated by an unpowered aircraft model can also be used in aerodynamic studies to improve the efficiency of the design. Testing encompasses both the 'clean' and 'dirty' configurations, without and with various aerodynamic control surfaces and the undercarriage deployed.

A series of tests were conducted in the tunnel during 2012 and 2013 to evaluate the acoustic signature of a new aircraft design. The signature of the model was detected by an acoustic, phased array of 97 microphones built into an 8.4 foot $(2.6 \mathrm{~m})$ diameter disk. The disk was mounted horizontally on a gantry in the ceiling space of the wind tunnel and could be driven to any location in the 22 feet by 50 feet area of the test section (Figure 3). The gantry motion was limited to a few centimetres per second and the noise sampling was carried out whilst the microphone array was stationary.

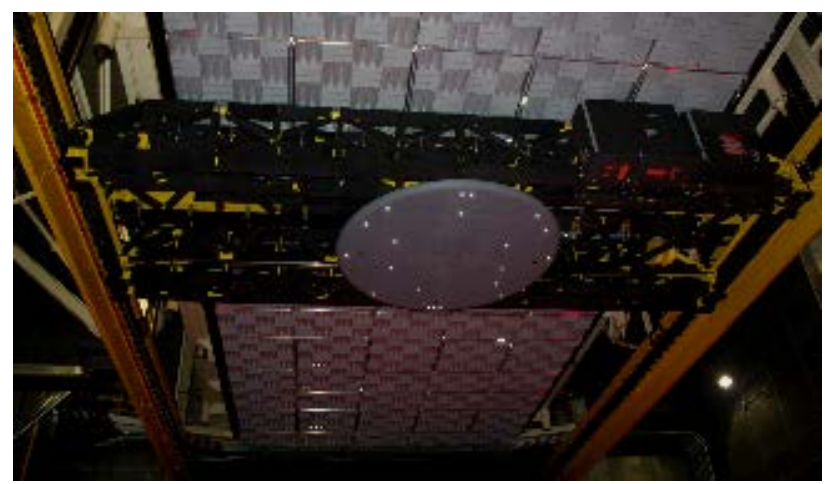

Figure 3. Microphone array disk mounted on the ceiling gantry in the $14 \times 22$ tunnel.

The task for the photogrammetric tracking was to determine the location of the centre of the microphone array to a precision of 1-2 mm. To enable the tracking of the centre of the array, 20 retro-reflective targets were attached to the surface and located using a GSI V-Stars ${ }^{4}$ system (see Figure 4). The V-Stars system achieved a coordinate precision of $0.03 \mathrm{~mm}$ using an INCA 3a camera and three $1.12 \mathrm{~m}$ Brunson bars (40 inch bars plus 2 inch offset targets) with a length precision of $0.05 \mathrm{~mm}$. The target array was then re-oriented onto the same datum as the microphone array based on the CAD locations of the microphones and a seven parameter conformal transformation.

The system developed to track the microphone array comprised eight IDS $\mu$ Eye 5480 cameras connected to a Cisco SG300-28P GigE switch with PoE. A Gardasoft Vision CC320 trigger timing controller was used to synchronously fire all eight cameras. The cameras have a 2560 by 1920 pixel, half inch format, CMOS sensor with a 2.2 micrometre pixel spacing. The $\mu$ Eye cameras record 16 bit images, of which $10-12$ bits are

${ }^{4}$ www.geodetic.com significant. The maximum acquisition rate for the cameras is 14 frames per second.

Whilst synchronisation of the cameras is not critical for the relatively slow transport speed of the microphone array, the trigger timing was tested using a laser light 'chopper' wheel at a rapid rotation speed. Visual comparisons of captured images of a retro-target on the wheel indicated that the synchronisation of the cameras is accurate to 2-3 milliseconds.

For the tests the cameras were fitted with $5 \mathrm{~mm}$ Koyo lenses to provide a wide angle of view. The focus rings of the lenses were taped to ensure no changes could take place. Retroreflective targets were used to provide the highest possible measurement precision and accuracy. To reduce the impact of ambient lighting in the tunnel, the cameras were fitted with red LED ring lights and matching $635 \mathrm{~nm}$ lens filters.

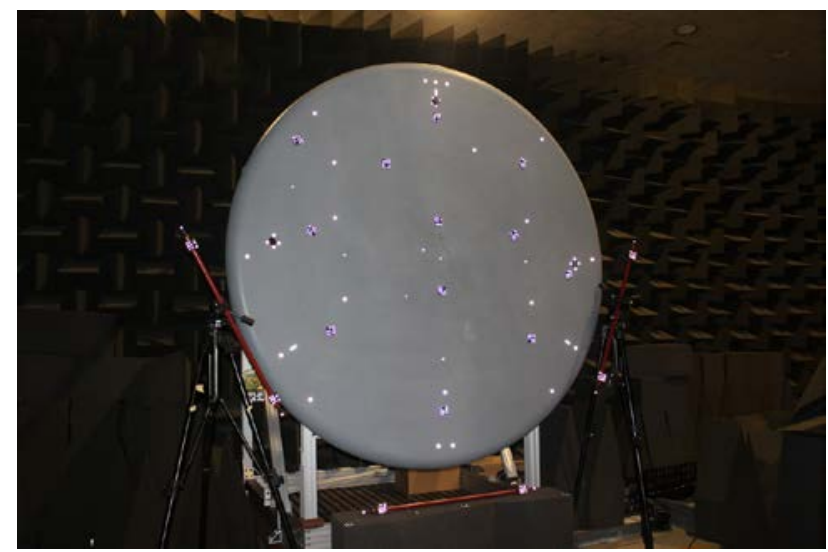

Figure 4. Set up for the coordination of the targets on the surface of the microphone array disk.

The eight cameras were calibrated in a laboratory using a portable calibration fixture. The cameras were mounted on tripods or fixed rails and the fixture was moved around within the fields of view of the cameras. Vision Measurement System $\left(\mathrm{VMS}^{5}\right)$ was used to process this very strong network of 70 targets and 400 exposures. The RMS image residual from the calibration was approximately 0.1 pixels.

The eight cameras were then placed on fixed rails at floor level in the tunnel, four on either side of the test section. Ball mount tripod heads were used to set the fields of view of the ceiling area. Two groups of four cameras each covered half of the test section area, with a small amount of overlap in the centre section. This geometry ensured every target on the microphone array would be imaged by a minimum of four cameras and the array could be tracked at all locations within the limits of the gantry movement.

Retro-reflective targets were placed on support structures in the ceiling to provide a fixed reference for the cameras. To introduce an accurate scale and align the targets with the tunnel coordinate system, six of the targets were coordinated using a Faro Xi V2 laser tracker with a single point precision of 0.03 $\mathrm{mm}$. The coordinates of the full array of reference targets, based on the tunnel coordinate system, were determined using the GSI V-Stars system. V-Stars coded targets were temporarily fixed to the ceiling structures to enable the automatic processing of the image measurements and network

${ }^{5}$ www.geomsoft.com/VMS 
computations. The fixed reference targets were located with a precision of $0.02 \mathrm{~mm}$ but the imperfect stability of the ceiling structures was expected to degrade the accuracy of the locations, especially in wind on test conditions.

This expectation was reflected in the resections of the eight cameras for initial, wind off tests. The resections typically produced RMS image residual errors ranging from 0.3 pixels to 0.8 pixels. Based on the resections, the intersections of the targets on the microphone array produced RMS image residual errors in the range of 0.5 to 1 pixel, and realised target coordinate precisions of approximately 2-4 mm. 6DoF transformations based on the 20 targets on the microphone array resulted in an RMS error typically in the range of $1 \mathrm{~mm}$ to 4 $\mathrm{mm}$ and a position precision of $0.2 \mathrm{~mm}$ to $0.5 \mathrm{~mm}$, which is within the required specification.

Contrary to expectations, very similar results were obtained with wind on testing. This outcome is partly due to the positioning of the microphone array and cameras out of the main air flow and the relatively low level of vibration generated within the test facility when operated at subsonic wind speeds.

Purpose-written software known as VMSCapture is used to capture and process the images. The graphic user interface displays live views from up to eight cameras, with a slider control to individually set the exposure time. VMSCapture captures 16 bit greyscale images either in a one-shot mode, in free-run mode where the image sets are captured continuously at the camera frame rate, or in external trigger signal mode where the cameras are fired using the hardware signal from the timing controller. One-shot mode generates TIFF images and is used primarily for exposure and resection testing for the initial set-up. Free run mode produces the most rapid acquisition of 34 frames per second, but the software-based trigger is not accurate. The hardware trigger mode does acquire accurately synchronised images but is limited to 1-2 frames per second. This modest frame rate is suitable for slow movement or a stopstart motion such as the microphone array.

VMSCapture has a number of modes of operation, based on an operator selection. The simplest modes simply log image files or 2D target image coordinates. Target images are identified using an edge detector, a circularity test and a target image span test. Any circular object within the field of view will be identified as a candidate, which is then confirmed based on a local threshold computation and an intensity weighted centroid within a small image patch centred on the candidate (Trinder, 1989). VMSCapture has the capability to simultaneously accept and analyse all eight 16 bit images synchronously captured by the $\mu$ Eye cameras.

3D mode includes a number of steps based on set-up information of the camera calibrations, camera positions and orientations, typically computed from resections, and target locations. The first step is a search amongst the candidate target images for fixed resection targets. If sufficient targets are identified then the resection of each camera can be updated. Next, the remaining target image candidates are searched for tracked targets. This search is based on the initialisation data for a new frame set, or the last frame set within a sequence. Any remaining target image candidates are then matched between cameras based on a tree-search correspondence solution (Hosseininaveh et al, 2013). Target coordinates are then computed based on intersection solutions and any locations outside of a 3D region of interest, in this case the bounds of the tunnel test section, are deleted.

For a $6 \mathrm{DoF}$ solution the final step is a 3D search for tracked targets. Automatic recognition using coded targets (Shortis and Seager, 2014) could not be used because of limitations on the available surface area of the disk. In the absence of coded targets, the first option is a search based on a previous frame set with a successful $6 \mathrm{DoF}$ computation. If there was no previous computation, or no successful computation, then the next option is to use the initial set-up data. The last option is a 3D search seeking the specific patterns of two and three target clusters on the microphone array disk (Figure 4). All of these searches are constrained to a specific 3D region of interest, such as that bounded by the limits of the gantry movement.

If none of the options were successful then the operator needed to re-initialise the software. This step was required on many occasions to recover from a poor fit to the target pattern on the disk computed by the 6DoF solution, caused by a partial mismatch of targets from the correspondence solution or the 3D search routine. In general, once the $6 \mathrm{DoF}$ solution 'lost lock' on the disk, the method would not recover without reinitialisation.

6DoF data and a time stamp for every frame set with a successful computation was transmitted via Ethernet to a tunnel control PC. This system acted as the master controller and data logger, collecting information from many systems and sensors using a variety of interfaces.

\subsection{Model Tracking in the National Transonic Facility}

The National Transonic Facility (NTF) at LaRC is the largest pressurised, cryogenic wind tunnel in the world and possesses unique capabilities to duplicate actual flight conditions. NTF provides the highest transonic Reynolds number (a measure of fluid flow similarity) testing capability in the world, and can use either air at temperatures up to $50^{\circ} \mathrm{C}$ or gaseous nitrogen at temperatures as low as $-150^{\circ} \mathrm{C}$ to achieve specific flight test conditions. With a wide range of customisable instrument and measurement techniques, both full-span (sting mounted) and semi-span (wall mounted) model testing is supported (Figure 2).

The $2.5 \mathrm{~m}$ high by $2.5 \mathrm{~m}$ wide by $7.6 \mathrm{~m}$ long test section of NTF is accessed through a removable wall section. The cameras and light sources in the ceiling, walls and floor are contained in 48 flush-mounted housings that view the test section through glass ports. The test section is sealed and inaccessible during wind on testing, but can be sealed and accessed through crawl-ways to allow calibration and set-up in wind off conditions.

A series of tests were conducted from 2012 to 2015 using the FastMac (Fundamental Aerodynamics Subsonic Transonic Modular Active Control) semi-span model. FastMac was used to both validate various tunnel systems and to investigate factors such as the effectiveness of circulation control to improve aerodynamic efficiency. The FastMac model has four air blowers that can reduce drag for subsonic and transonic cruise conditions.

During the FastMac testing in 2015 the opportunity was taken to commence the progressive replacement of older CameraLink interface cameras with IDS $\mu$ Eye GigE cameras. The NTF test section typically has 6-8 cameras capturing images, depending on the test configuration. The remaining ports are used for light 
sources to illuminate the targets. As a transition, four IDS $\mu$ Eye GigE cameras were installed, two in the ceiling and two in the removable wall, to enable the GigE and CameraLink systems to operate in parallel or independently. $\mu$ Eye 6280 cameras with CCD image sensors were selected to take advantage of a larger pixel size of $3.45 \mu \mathrm{m}$ and an improved light sensitivity. The image resolution of 2448 by 2050 pixels is similar to the IDS 5480 cameras used in the $14 \times 22$ tunnel, whilst the maximum acquisition rate of 8 frames per second is slower but not a limitation for NTF. The GigE cameras were again connected to an Ethernet switch and a timing controller. Power is provided to each camera individually through a switched environmental control system that senses the camera temperature. Cameras are automatically shut down if the temperature exceeds the limit specified by the manufacturer.

The fundamental measurements required for aerospace models in wind tunnels are the determination of model pitch, also known as angle of attack (AoA), and the deformation of the flight surfaces (Burner and Martinson, 1996). For semi-span models such as FastMac, the half aircraft model is attached to a rotational stage built into the tunnel wall. The AoA of the stage is set by an electric motor and sensed by a rotary encoder. However the rotary encoder may be subject to bias and drift, so an independent measurement of AoA is essential for this critical parameter.

As the AoA is varied through a series of take off, cruise and landing configurations, the deformation of the flight surfaces is measured to correlate with aerodynamic factors such as lift and drag. The deformation evaluation has two components, wing deflections and wing twist. Wing deflection and wing twist are defined as the change in the location and change in the orientation of the aerodynamically loaded surface relative to the unloaded surface.

To measure the AoA and deformations during testing, the cameras must be calibrated and the test section set-up must be carried out. In this case the cameras were calibrated in-situ using a 3D 'step block'. VMS was used to process this very strong network of 117 targets and 90 exposures. The RMS image residual of 0.05 pixels from this calibration is an improvement compared to previous calibrations of $\mu$ Eye cameras.

There are significant differences between the set-up of the cameras in the $14 \times 22$ tunnel and NTF. To optimise the fields of view of the semi-span model (see Figure 5), the cameras were fitted with $9 \mathrm{~mm}$ and $15 \mathrm{~mm}$ lenses for the ceiling and wall cameras respectively. The $9 \mathrm{~mm}$ lenses in particular have a substantive radial lens distortion profile compared to a low profile for the $5 \mathrm{~mm}$ lenses. At a radial distance of $3 \mathrm{~mm}$, the approximate magnitude of the distortion is $10 \mu \mathrm{m}, 75 \mu \mathrm{m}$ and $25 \mu \mathrm{m}$ for the $4 \mathrm{~mm}, 9 \mathrm{~mm}$ and $15 \mathrm{~mm}$ lenses respectively. The calibration of the cameras at NTF includes the refractive effects of the glass camera ports. The combination of larger distortion magnitudes plus the effects of refraction and non-uniformities from the ports would be expected to degrade the quality of the image measurements in NTF.

However the other significant difference between the cameras used in the $14 \times 22$ tunnel and NTF is that the $\mu$ Eye cameras in NTF were operated in 2 by 2 binned mode to enhance the intensity of the image. Operation in this mode is preferred based on the current lighting levels in NTF, and the need for a relatively fast exposure time to limit the impact of tunnel vibration. Binning effectively increases the pixel size to $6.9 \mu \mathrm{m}$ and decreases the image resolution to 1224 by 1024 pixels. Although a more thorough comparison test based around an analysis of image quality and imaging network geometry is warranted to validate these results, the advantage of the improved image quality from the binning process appears to outweigh the other factors.

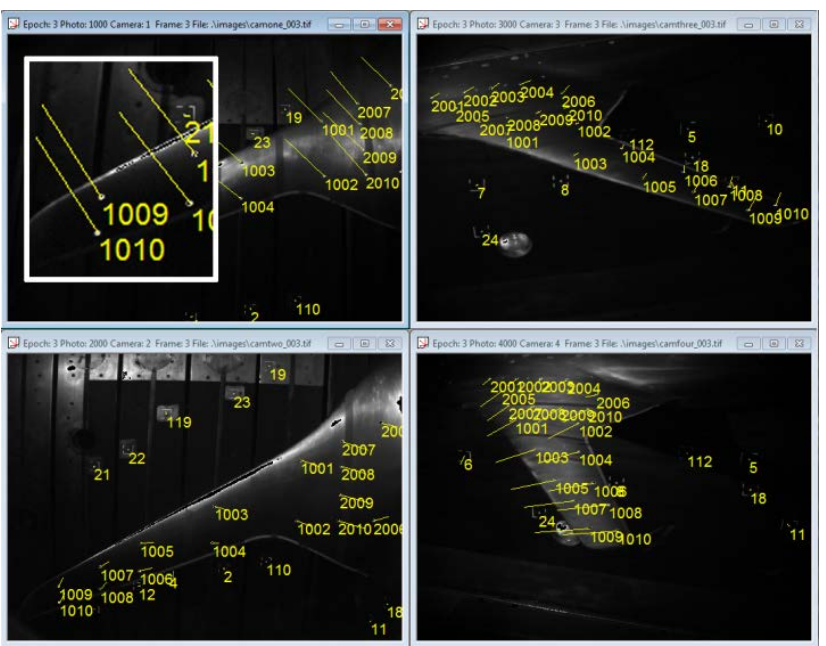

Figure 5. Camera views of a semi-span model and intersection errors for a polar sweep validation in NTF (detail view inset).

The set-up in the tunnel test section requires the model to be placed into the zero AoA position and the photogrammetric network to be aligned to the tunnel coordinate system. For the NTF test section the datum is the centre point of the rotation stage and the $\mathrm{X}$-axis is aligned with the centre-line of the tunnel. Because the model obscures the rotation stage, the datum is inferred from targets placed on the walls and floor of the tunnel test section. These targets are adopted using planes of best fit to align the set-up network with the tunnel coordinate system. Targets are also placed at the edge of the rotation stage so that the obscured pivot point can be located.

Simultaneously with the set-up of the new camera system, experiments were underway with different target options and lighting of the NTF test section. Requirements for targeting in NTF are challenging. The targets must be fixed to the test section with no possibility of breaking loose in wind on conditions, and must withstand a temperature range of $-150^{\circ} \mathrm{C}$ to $+50^{\circ} \mathrm{C}$. The surface finish of most models is required to be 10 micro-inches (0.25 micrometres) or better with no forward facing steps on the wing surface to interfere with the flow. These characteristics are not compatible with retro targeting materials. In general, ambient light targets have been used in preference to retro-reflective targets for these reasons.

As noted, passive targets have the major disadvantage that, as the AoA of the model changes, variation in lighting intensity and glints from shiny metallic surfaces prevent the targets from being measured (Figure 1). To overcome this limitation and provide a target image similar in quality to retro-reflective targets, a new scheme of fluorescent paint and ultra-violet lighting was implemented in the NTF test section (Figure 5). Using templates, the fluorescent target solution also permitted coded targets (Shortis and Seager, 2014) to be included as fixed reference targets and thereby permit the automated processing of resections. 
The set-up in the tunnel test section was imaged using a Canon Powershot A80 digital camera with a sensor resolution of 2272 by 1704 pixels. The network of 30 exposures and 40 targets was processed using VMS. The RMS image residual was approximately 0.2 pixels and the average precision of the $\mathrm{XYZ}$ target coordinates was $0.1 \mathrm{~mm}$. After fine tuning of the lighting and re-painting of some targets, a second network realised an improved RMS image residual of 0.1 pixels and an average precision of the XYZ target coordinates of $0.05 \mathrm{~mm}$.

Validation of the set-up is conducted using polar sweep tests, comprising measurements of static AoA settings in wind off conditions whilst the model is rotated by $1^{\circ}$ increments. In the polar sweep tests, the camera resections typically produced RMS image residual errors ranging from 0.1 pixels to 0.3 pixels. Based on the resections, the intersections of the targets on the wing of the semi-span model produced RMS image residual errors in the range of 1 to 4 pixels for all four cameras. The patterns of image residuals for four cameras clearly indicate the presence of systematic errors (Figure 5). The magnitudes and patterns of the image residuals were similar for wind off, wind on and cryogenic wind on tests.

In comparison, RMS image residuals of less than one pixel resulted for the two pairs of cameras in the ceiling and the wall section when processed independently. The patterns of residuals tended to be more random, but systematic errors were still evident.

The apparent errors can be attributed to three possible sources. First, the two cameras mounted in the wall are part of the structure that is removed to gain access to the test section, potentially leading to relocation or disturbance of the camera positions and orientations. Continuous resection using the reference targets on the walls and ceiling do not eliminate these systematic errors, indicating an inconsistency between the calibration of the cameras and the initial set-up in the test section of the tunnel.

Second, all camera housings are heated, but for logistics reasons only the wall housings are cooled. The temperatures in the housings vary dependent on radiant heat from the cameras and the temperature of the flow in the tunnel. The CCD sensors operate at a higher temperature than the CMOS sensors and can exceed the recommended limit when the tunnel is operating with air at $50^{\circ} \mathrm{C}$. The ceiling cameras are automatically shut down if they exceed the temperature limit. Continual overheating followed by a cooling down period has an unknown impact on both calibration and orientation. In the future the CCD based cameras will be replaced by the CMOS cameras to reduce the effect of self-heating.

The temperatures of the nitrogen or air flowing through the test section and various structures in the NTF wind tunnel are monitored in real time. However it is worthy of note that the models generally do not include temperature sensors. Models are constructed from very stable Vascomax 300 or Nitronic 40 stainless steel which has a thermal expansion coefficient in the range of 5 to 10 parts per million.

Finally, there are significant levels of vibration when the tunnel is operating in wind on mode at transonic speeds. The short term effects of the vibration can be remedied by multiple image averaging for semi-span models. However the impact of vibration in the longer term, combined with the extremes of operating temperatures, is unknown. Sting mounted models are subject to greater magnitudes of vibration compared to semi- span models and require more sophisticated noise suppression techniques.

Despite these errors, the intersections realised target coordinate precisions of approximately $0.1 \mathrm{~mm}$. 6DoF transformations based on the 10 targets on the fuselage and wing root of the model resulted in an RMS error typically in the range of $0.5 \mathrm{~mm}$ to $1 \mathrm{~mm}$. An estimated precision of the AoA of $0.1^{\circ}$ is based on the $6 \mathrm{DoF}$ computations using the 10 targets. A comparison between the rotation stage data and the $6 \mathrm{DoF}$ data for a typical polar sweep sequence shows a consistent offset that ranges between $0.01^{\circ}$ and $0.1^{\circ}$. The source of this discrepancy requires further testing and is further evidence of an inconsistency in the calibration and set-up data sets. However the offset is most likely caused by a combination of bias in the rotation stage and misalignment between the reference target coordinate system and the tunnel coordinate system.

\section{CONCLUSIONS}

Clearly there is scope for further testing and further improvement in the video tracking systems at LaRC. In particular, the systematic errors experienced in the polar sweep validations in NTF need to be modelled or eliminated to ensure the highest levels of achievable accuracy. Further testing and analysis of the wing deflection and twist measurements is necessary to validate the results.

Notwithstanding these considerations, measurement of aerospace models in wind tunnel test sections are novel and specialised measurement tasks for which close range photogrammetry is well suited. No other solution can provide surface or $6 \mathrm{DoF}$ data which is as flexible, accurate and reliable, and incorporates directly computed uncertainty limits. The video measurement systems developed at LaRC are considered to be mature, because the equipment is reliable, the methodologies are well practised and the algorithms are robust. However it is clear that the systems will continue to be further developed, especially in terms of further automation of measurement processes and adopting new imaging technologies.

\section{REFERENCES}

Barrows, D. A., 2007. Videogrammetric model deformation measurement technique for wind tunnel applications. $45^{\text {th }}$ AIAA Aerospace Sciences Meeting and Exhibit, Reno, Nevada, USA. Paper 1163.

Burner, A. W. and Martinson, S. D., 1996. Automated wing twist and bending measurements under dynamic load. Proceedings, $19^{\text {th }}$ AIAA Advanced Measurement and Ground Testing Technology Conference, New Orleans, USA. Paper 962253.

Burner, A. W., Snow, W. L. and Goad, W. K., 1985. Close range photogrammetry with video cameras. Proceedings, Technical Papers, 51st American Society of Photogrammetry Annual Meeting, Washington, U.S.A., pp. 62-77.

Childers, B. A., Snow, W. L., Jones, S. B., Franke, J. M. and Shortis, M. R., 1994. Support of wake vortex detection research in flight and wind tunnel testing using videometric techniques. International Archives of Photogrammetry and Remote Sensing, Vol. 30 (5), pp 41-46.

Childers, B. A., Snow, W. L. and Shortis, M. R., 1994. Videometric system using VITC for computer-aided image 
management. Videometrics III, SPIE Vol. 2350, S. F. El-Hakim (Ed.), pp 93-98.

Cohen, S. and Hockaday, S., 1998. A concept paper for separation safety modelling - FAA/Eurocontrol Cooperative Effort on Air Traffic Modeling for Separation Standards. Report to Federal Aviation Administration and Eurocontrol. 178 pages.

Graves, S. G. and Burner A. W., 2001. Development of an intelligent videogrammetric wind tunnel measurement system. Conference on Optical Diagnostics for Fluids, Solids and Combustion, SPIE Vol. 4448, pp 120-131.

Pappa, R., Black, J. T., Blandino, J. R., Jones, T. W., Danehy, P. M., and Dorrington, A. A., 2003. Dot-projection photogrammetry and videogrammetry of gossamer space structures. Journal of Spacecraft and Rockets, 40(6), 858-867.

Pappa, R. S., Giersch, L. R., and Quagliaroli, J. M., 2001. Photogrammetry of a $5 \mathrm{~m}$ inflatable space antenna with consumer-grade digital cameras. Experimental Techniques, Society for Experimental Mechanics, 25(4): 21-29.

Pappa, R. S., Jones, T. W., Black, J. T., Walford, A., Robson, S. and Shortis, M. R., 2002. Photogrammetry methodology development for gossamer spacecraft structures. Sound and Vibration, August, pp 12-21.

Hosseininaveh, A., Robson, S, Boehm, J. and Shortis, M. R., 2013. Image selection in photogrammetric multi-view stereo methods for metric and complete 3D reconstruction. Videometrics, Range Imaging, and Applications XII, SPIE Vol. 8791, F. Remondino and M. R. Shortis (Editors), paper 07. 11 pages.

Robson, S. and Shortis, M. R., 1997. Surface characterisation by tracking discrete targets. Fourth Conference on Optical 3-D Measurement Techniques, A. Gruen and H. Kahmen (Editors), Wichmann, pp 179-186.

Shortis, M. R., 1989. Industrial photogrammetry at the NASA Langley Research Center. Proceedings, Symposium on Surveillance and Monitoring Surveys, University of Melbourne, Australia, pp. 218-231.

Shortis, M. R., 2015. Calibration techniques for accurate measurements by underwater camera systems. Sensors 15(12): 30810-30826; doi: 10.3390/s151229831

Shortis, M. R., Robson, S. and Beyer, H. A., 1998. Principal point behaviour and calibration parameter models for Kodak DCS cameras. The Photogrammetric Record, 16(92): 165-186.

Shortis M. R., Robson, S., Pappa, R. S., Jones, T. W. and Goad, W. K., 2002. Characterisation and tracking of membrane surfaces at NASA Langley Research Center. International Archives of Photogrammetry and Remote Sensing, 34(5): 90-94. ISSN 1682-1777.

Shortis, M. R. and Seager, J. W., 2014. A practical target recognition system for close range photogrammetry. The Photogrammetric Record, 29(147): 337-355.

Shortis, M. R. and Snow, W. L., 1995. Calibration of CCD cameras for field and frame capture modes. Conference on Digital Photogrammetry and Remote Sensing '95, SPIE Vol. 2646, pp. 2-14.

Shortis, M. R. and Snow, W. L., 1997. Videometric tracking of wind tunnel aerospace models at NASA Langley Research Centre. The Photogrammetric Record, 15(85): 673-689.
Shortis, M. R., Snow, W. L., Childers, B. A. and Goad, W. K., 1993. The influence of storage media on the accuracy and repeatability of photogrammetric measurements using CCD cameras. Videometrics II, SPIE Vol. 2067, S. F. El-Hakim (Ed.), pp 80-92.

Shortis, M. R., Robson, S., Jones, T. W. and Lunsford, C. B. 2007. Parachute model validation using image sequences. Eighth Conference on Optical 3-D Measurement Techniques, A. Grun and H. Kahmen (Eds), ETH Zurich, Switzerland, ISBN 3906467-67-8, Volume I, pp 72-79.

Snow, W. L., Childers, B. A., Jones, S. B. and Fremaux, C. M., 1992. Recent experiences with implementing a video based six degrees of freedom measurement system for airplane models in a 20 foot diameter vertical spin tunnel. Videometrics, SPIE Vol. 1820, S. F. El-Hakim (Ed.), pp. 158-180.

Snow, W. L. and Shortis, M. R., 1995. A rigid body motion analysis system for offline processing of time coded video sequences. Videometrics IV, SPIE Vol. 2598, S. F. El-Hakim (Ed.), pp 220-232.

Trinder, J. C., 1989, Precision of digital target location. Photogrammetric Engineering and Remote Sensing, 55(6): 883886.

Wiora, G., Babrou, P. and Männer, R., 2004. Real time high speed measurement of photogrammetric targets. In: Pattern Recognition. C. Rasmussen, H. Bülthoff, B. Schölkopf and M. Giese, Springer Berlin Heidelberg. 3175: 562-569. 